\title{
Subcellular carrier-based optical ion-selective nanosensors
}

\author{
Susana Carregal-Romero ${ }^{\dagger}$, Jose-Maria Montenegro $^{\dagger}{ }$, Wolfgang J. Parak and Pilar Rivera_Gil*
}

Department of Biophotonics, Institute of Physics and WZMW, Philipps University of Marburg, Marburg, Germany

Edited by:

Amalia M. Dolga, Philipps-Universität Marburg, Germany

Reviewed by:

Jason B. Wu, Cedars-Sinai Medical

Center, USA

Loretta Laureana Del Mercato,

National Research Council of Italy,

Italy

*Correspondence:

Pilar Rivera_Gil, Department of

Biophotonics, Institute of Physics and

WZMW, Philipps University of

Marburg, Renthof 7, D-35037

Marburg, Germany.

e-mail: pilar.riveragil@

physik.uni-marburg.de

${ }^{+}$Susana Carregal-Romero and

Jose-Maria Montenegro have

contributed equally to this work.
In this review, two carrier systems based on nanotechnology for real-time sensing of biologically relevant analytes (ions or other biological molecules) inside cells in a non-invasive way are discussed. One system is based on inorganic nanoparticles with an organic coating, whereas the second system is based on organic microcapsules. The sensor molecules presented within this work use an optical read-out. Due to the different physicochemical properties, both sensors show distinctive geometries that directly affect their internalization patterns. The nanoparticles carry the sensor molecule attached to their surfaces whereas the microcapsules encapsulate the sensor within their cavities. Their different size (nano and micro) enable each sensors to locate in different cellular regions. For example, the nanoparticles are mostly found in endolysosomal compartments but the microcapsules are rather found in phagolysosomal vesicles. Thus, allowing creating a tool of sensors that sense differently. Both sensor systems enable to measure ratiometrically however, only the microcapsules have the unique ability of multiplexing. At the end, an outlook on how more sophisticated sensors can be created by confining the nano-scaled sensors within the microcapsules will be given.

Keywords: molecular imaging, cells, non-invasive, ions, sensors, colloidal nanoparticles, polyelectrolyte multilayer capsules, optical read-out

\section{INTRODUCTION}

Control of intracellular ion homeostasis is essential for all cellular organisms. At physiological condition ions like $\mathrm{K}^{+}$or $\mathrm{H}^{+}$ are found at high concentrations inside the cells, whereas ions like $\mathrm{Na}^{+}, \mathrm{Ca}^{2+}$, or $\mathrm{Cl}^{-}$are rather found extracellularly (Lang, 2007). Many pathological situations like liver insufficiency, diabetic ketoacidosis, hypercatabolism, fibrosing disorders, sickle cell anemia, infection (Lang, 2007), cystic fibrosis (Stutts et al., 1995; Mall et al., 1996; Matsui et al., 1998), or intoxication with metals (Darbha et al., 2007) are associated with a defective regulation of the ion concentrations. For example, disregulation of the $\mathrm{K}^{+}$entry might alter $\mathrm{Ca}^{2+}$ homeostasis; thus, leading to neuronal degeneration and all associated diseases like Alzheimer, stroke or ischemia, and epilepsy (Dolga et al., 2011).

So far most of the techniques employed to measure ion concentrations make use of electrodes (Bakker et al., 1997) or fiber-based optodes (Tan et al., 1992; Buhlmann et al., 1998). These systems work well for solutions but in general not for cellular organisms, since they are too big to enter the cells over extended periods of time. There are other techniques like microanalysis that can measure intracellular analyte concentrations. However they are destructive for the biological sample (Cameron et al., 1980). For subcellular (intracellular) analyte detection smaller non-invasive sensors are required, especially if long term measurements in live cells are envisaged. One possibility toward this direction is the use of particles as carrier matrix/container for analyte-sensitive molecules. Examples of such nano/micrometer-sized containers include solid particle matrices (Burns et al., 2006; Hidalgo et al., 2009), liposomes (Nguyen and Rosenzweig, 2002), hollow fiber membranes (Ballerstadt and Schultz, 2000), vesicles (Dimosthenis and Athanasios, 2011), and polyelectrolyte capsules (Kreft et al., 2007; Rivera Gil et al., 2008; Delcea et al., 2010). Carriers must be designed in a way that ions or molecules are able to freely diffuse to the location of the sensor. The sensors allow for the transduction of chemical concentration information into a readout signal. Mostly optical signals are used as read-out because of their ease of detection. However, more sophisticated and sensitive nanosensors which make use of shift in the plasmon peak of gold nanoparticles upon the close presence of analytes have also been described (Alvarez-Puebla et al., 2009; Rodriguez-Lorenzo et al., 2011; Perez-Pineiro et al., 2012).

In this review, we discuss two of such systems for the time resolved optical measurement over extended periods of time of the analytes concentration (ions or other (small) biological molecules) inside cells in a non-invasive way. One system, the so-called nanoparticle system, is based on hybrid materials (inorganic and organic) and has a size in the nanometer scale. The second system, the polymer capsules, is composed out of organic materials and has a size in the micrometer scale range. In both cases, nano-/microparticles act as carrier for the sensor molecules (conventional organic analyte-sensitive fluorophores) and exhibit an optical read-out. Hereby the sensing ability is based on changes in the photoluminescence emission subsequent to ion binding in a reversible manner. In case two types of fluorophores (one sensitive and one insensitive to the analyte) are incorporated in the system, ratiometric measurements can be performed. In this way differences in the read-out due to quenching, loading efficiency, or degradation of the analyte-sensitive fluorophore are partly circumvented. Analogously, both systems (nano- and microparticles) can carry the same sensor molecules. Since these two systems differ 
from each other in size and chemical composition, their uptake pathway by cells and their intracellular location can be different. Therefore, different information from the intracellular environment, where each sensor is preferentially located can be obtained. In this way, depending on the localization of the analyte to be determined one can make use of the nano- or the micro-sensors.

In this review, the geometry as well as the advantages and disadvantages of both sensors (nano/micro) will be first discussed. Afterward, how both systems can complement each other to create a more sophisticated sensor, which includes the functionalities of both single sensing systems will be presented.

\section{ION SENSORS AT THE NANO-SCALE}

In the last years, nanotechnology has enabled to create new materials with a wide variety of applications being the bio-medical field the most promising (Cui et al., 2007; De et al., 2008; PeteiroCartelle et al., 2009; Rivera_Gil et al., 2010; Thanh and Green, 2010). Nanomaterials are engineered in a submicron size range of a few to a few hundreds of nanometers which are on purpose designed to result in new biological/medical effects due to their unique physicochemical properties that differ from their macroscopic counterparts. Nanoparticles tailored for biological applications are commonly composed of an inorganic core and a biocompatible surface coating that provides chemical stability under physiological conditions, colloidal dispersibility in aqueous solution, and reduced toxicity (Pellegrino et al., 2004). The surface coating can be chemically modified with different molecules to introduce additional functionalities to the nanomaterial (Lin et al., 2008). Within other examples, analyte (ion)sensitive fluorophores can be covalently linked to the outer shell of the nanoparticles to obtain nano-structured sensing systems (Figure 1A; Brown and McShane, 2005). Furthermore, other kinds of molecules can additionally be attached to the nanoparticle surfaces. This can be used to create ratiometric nano-scale sensors. For this purpose, non-sensitive fluorophores, which do not respond to concentration changes of the analyte of study can be co-attached together with analyte-sensitive fluorophores to the nanoparticles surface (Riedinger et al., 2010; Zhang et al., 2010). In this way, analyte-sensitive fluorophores as well as nonsensitive fluorophores are located at the same nanoparticle and the analyte concentration can be determined as the ratio of the fluorescence read-out of both fluorophores. If fluorescent semiconductor nanoparticles (quantum dots) are used as carrier for the analyte-sensitive fluorophores, the intrinsic nanoparticle fluorescence can be used as reference for ratiometric analysis too. Following this scheme, nanoparticles sensitive to ions like chloride $\left(\mathrm{Cl}^{-}\right.$; Riedinger et al., 2010), protons $\left(\mathrm{H}^{+}\right.$; Zhang et al., 2010), sodium $\left(\mathrm{Na}^{+}\right)$, or potassium $\left(\mathrm{K}^{+}\right)$, can be efficiently produced. Such systems have already been applied for detecting concentrations of ions inside living cells (Zhang et al., 2011; Rivera_Gil et al., 2012).

There are several considerations to keep in mind when designing a nanoparticle-based sensor. Depending on the nanomaterial used as carrier, it is possible to find different unwanted interactions with the read-out. Thus if metallic nanoparticles (gold, silver) are used, fluorescence quenching can be observed (Dulkeith et al., 2002; Fu and Lakowicz, 2009; Zhu et al., 2010). On the other side,

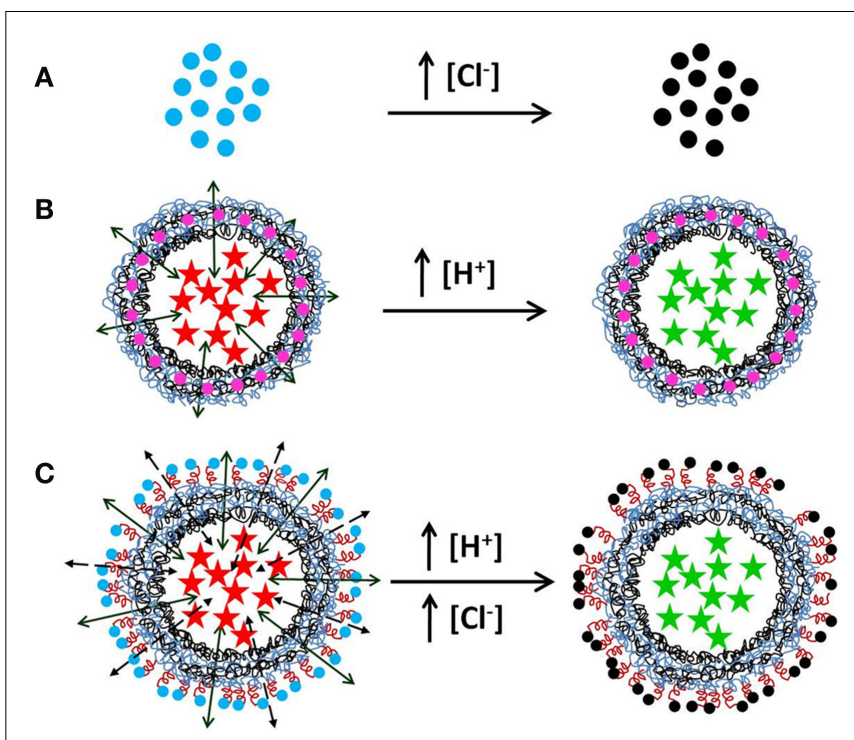

FIGURE 1 | Nanoparticle-modified multifunctional sensor capsules. A schematic illustration of the three proposed ion sensor systems is hereby illustrated. (A) Nanoparticles functionalized with an ion-sensitive fluorophore, e.g., with Amino-MQAE, (2-[2-(6-methoxyquinoliniumchloride) ethoxy]-ethanamine hydrochloride), which is a chloride $\left(\mathrm{Cl}^{-}\right)$sensitive fluorophore. The nanosensors will show blue fluorescence (blue dots) at low concentration $\left(\left[\mathrm{Cl}^{-}\right]\right.$) but the fluorescence will be quenched (black dots) in the presence of a high $\left[\mathrm{Cl}^{-}\right]$in the environment of the nanoparticles. (B) Polyelectrolyte microcapsules loaded with an ion-sensitive fluorophore, e.g., with $\mathrm{SNARF} \circledast$, which is sensitive to protons $\left(\mathrm{H}^{+}\right)$. Its fluorescence emission shifts from red (red stars) to green/yellow (green stars) by increasing the concentration of $\mathrm{H}^{+}$in the environment of the polyelectrolyte microcapsule. The wall is modified with an ion-insensitive dye, e.g., Quantum dots or organic dyes (pink dots) for multiplexed measurements. (C) Multisensing polyelectrolyte microcapsule made by embedding ion sensor fluorophores in the inner cavity of the hollow capsule and nanoparticles functionalized with ion-sensitive fluorophores in the wall. Notice that in order to avoid screening of the nanoparticle-based fluorophores by counter-ions the nanoparticle must be separated from the charged surface of the capsule (for example with a PEG molecule). In the figure, the capsule cavity is loaded with SNARF and the outer shell is conjugated to chloride-sensitive nanoparticles. These figures have not been drawn to scale.

the use of semiconductor fluorescent nanoparticles can lead to fluorescence resonance energy transfer (FRET), which in turn can be a desired process to create more sophisticated sensors (Suzuki et al., 2008; Yakovlev et al., 2009). Moreover, to maintain the colloidal stability of these nanoparticles in aqueous solution, either steric or electrostatic repulsion between the nanoparticles is required. In the case of electrostatic repulsion the nanoparticles need to bear a big net charge. This net charge produces a local environment on the nanoparticle surface, which is different from bulk solution. This is due to the fact that each charged nanoparticle attracts a cloud of counter-ions. In this way close to the nanoparticle surface the concentration of oppositely charged ions is increased, whereas the concentration of ions of the same charge is decreased, as described in first order by the Debye-Hückel theory (Debye and Hückel, 1923a,b). Thus the read-out of ion-sensitive fluorophores close to the nanoparticle surface does not reflect the real ionic concentration in the bulk solution. This limitation can be partially solved 
by modifying the linkage of the fluorophore to the nanoparticle. The use of polymeric spacers based on poly(ethylene)glycol (PEG) molecules has been reported to be an useful tool to modify the distance between the nanoparticle and the fluorophore, thus bringing the ion-sensitive fluorophores to distances further away from the nanoparticle surface where the ion concentration is more similar to the bulk concentration (Riedinger et al., 2010; Zhang et al., 2010). Nevertheless, the local net charge present at the nanoparticle surface can also be used to tune the sensitivity of analyte-sensitive fluorophores to make them useful for physiological conditions. For example, oregon green is a commercial acidic $\mathrm{pH}$ fluorescence indicator with a $p K_{\mathrm{a}}$ of $\sim 4.7$, i.e., the range of $\mathrm{pH}$ detection goes from $\sim \mathrm{pH} 3.5$ to 6 . This means that for applications where a $p K_{\mathrm{a}}$ around 7 is desired, this $\mathrm{pH}$-sensitive fluorophore is not useful. Making use of Debye-Hückel screening on charged nanoparticle surfaces the effective $p K_{\mathrm{a}}$ of oregon green can be tuned from 4.7 (free oregon green) to 7.9 for oregon green linked to the surface of negatively charged nanoparticles (Zhang et al., 2011).

There are also some other considerations to keep in mind when using nanoparticles as carriers for the sensor molecules especially for biological applications. In this case like in other kinds of sensors (Grabchev et al., 2004; Aranzaes et al., 2006; Grabchev and Guittonneau, 2006), the analyte-sensitive fluorophore is located in the surface of the nanoparticle, being therefore unprotected from the environment and sensitive to degradation. Degradation can occur either enzymatically, by disrupting the structure of the sensor or chemically, due to irreversible damage of the fluorescence signal, e.g., by oxidation. Both cases are related and not exclusive and at any rate result in the disruption of the sensor signal. Although degradation will not occur within minutes or hours and measurements over short periods of time (enough for most relevant bio-medical applications) can still be accurately performed, for long term measurements, i.e., over days this fact should be kept in mind.

One of the big advantages of the nanoparticle-based sensors is that their geometry can be tuned to direct them to specific intracellular locations. Physicochemical parameters of the nanoparticles like colloidal stability, size, and surface properties are key factors that have been named to influence the cellular behavior of nano-scale particles (Mailander and Landfester, 2009). For example, the surface charge clearly manipulates the overall uptake rate of nanoparticles and their pathway of internalization (HarushFrenkel et al., 2008; Ge et al., 2009; Chung et al., 2010). There are some nanoparticle systems in which positively charged particles in contrast to negatively charged particles are not found in the endosomes immediately after cellular uptake, though particles of both charges are found in lysosomal structures after $24 \mathrm{~h}$ (Schweiger et al., submitted). By changing other physicochemical parameters like size and shape, different endocytosis mechanisms, and thus locations can be observed (Nakai et al., 2003; Osaki et al., 2004). For example, colloidal gold nanoparticles modified with ligands that utilize the membrane receptor ErbB2 to enter the cell showed a location within multivesicular structures or within endosomes depending on their size (Jiang et al., 2008). Moreover, signal-mediated transport via pores and thus nuclear and cytoplasmatic localization has been proposed for inhaled ultrathin $(<0.1 \mu \mathrm{m})$ nanoparticles (Geiser et al., 2005). Quantum dots can also be engineered to locate in synaptic vesicles of cultured neurons. Their $\mathrm{pH}$-dependent fluorescent signal enabled long term tracking of the synaptic activity in situ (Zhang et al., 2009). More sophisticated tuning processes include the surface decoration with functional groups that can direct the nanoparticles to specific intracellular locations. A quantum dot-peptide conjugate that carries the SV40 large T antigen nuclear localization signal has also been described to deliver these nanoparticles to the nucleus of living cells (Chen and Gerion, 2004). Gold nanoparticles stabilized with the pentapeptide CALNN or a combination of two different cell penetrating peptides was partially found freely dispersed in the cytosol (Nativo et al., 2008). Small engineered quantum dots fused with the GluR2 subunit of the AMPA-type glutamate receptor, which localizes to postsynaptic membranes, significantly improved the access to and accumulation in the neuronal synapses (Howarth et al., 2008).

In conclusion, the use of nanoparticles as carriers for ionsensitive fluorophores shows several advantages. For example, as many fluorophores can be linked per nanoparticle, the detection efficiency increases significantly. Furthermore, the incorporation of the molecular sensors to the nanoparticles avoids problems associated with the molecules alone, like delocalization or various non-specific interactions. Since the different nanoparticles accumulate in specific subcellular compartments, the read-out of the sensor is highly localized and can be easily detected by fluorescence microscopy. By changing physicochemical parameters like shape, size, surface chemistry, and charge, the intracellular fate of the nanoparticle-based sensor can controllably be tuned. In this way, the nanosensors can be targeted to specific compartments where the concentration of analytes has to be determined.

\section{ION SENSORS AT THE MICRO SCALE}

Due to the high surface-to-volume ratio of nanoparticles analytesensitive fluorophores can be attached to their surface. Such geometry would not warrant for optical loading of micrometersized carriers though. For micrometer-sized carriers, the loading capacity can be enhanced by filling their whole volume with analyte-sensitive fluorophores, thus reducing the quantity of sensors used. For such purpose the carriers must be designed in a way that ions or molecules are able to freely diffuse into the container, while compartmentalizing the sensing reagents (i.e., the analyte-sensitive fluorophores) in the volume. The optical sensors as described before are often organic fluorescent dyes that can reversibly change their fluorescence emission depending on the presence of a specific analyte. This section is focused on the application of polyelectrolyte multilayer capsules as carrier system, since they are one of the most versatile platforms of micrometersized containers (Choi et al., 2007; De_Geest et al., 2009; Becker et al., 2010). Furthermore, they have been proved to be excellent carriers for different cargoes to different cells in vitro and in vivo as well as exhibiting enhanced biocompatibility (De_Koker et al., 2007; Hartig et al., 2007; Rivera_Gil et al., 2009; Kolbe et al., 2011). Capsules carrying sensor molecules have been recently synthesized to detect biologically relevant ions such as $\mathrm{K}^{+}, \mathrm{Na}^{+}, \mathrm{H}^{+}$, and $\mathrm{Cl}^{-}$ (del_Mercato et al., 2011). Moreover, due to their porosity the capsules can serve as biosensors for bigger molecules like glucose by entrapping enzymatic proteins or engineered binding proteins 
(McShane and Ritter, 2010; Kazakova et al., 2011). The synthesis of capsules is based on layer-by-layer self-assembly in which polyelectrolytes of different charges are deposited in an alternating sequence onto a sacrificial template (Donath et al., 1998; Sukhorukov et al., 1998). The versatility of this synthetic protocol makes it possible to produce capsules of different compositions (biodegradable $v s$. non-degradable, etc.) with a huge range of different properties ( $\mathrm{pH}-$-, temperature-sensitivity, etc.; Rivera_Gil et al., 2009; Stuart et al., 2010). There are two main procedures to load the capsule cavity with macromolecules: (i) the coprecipitation of the cargo (in our case the analyte-sensitive fluorophores) during the template formation, or (ii) the "postloading" of empty capsules taking advantage of the capsule volume decrease due to environmental changes (temperature, $\mathrm{pH}$, ionic strength; Becker et al., 2010). The coprecipitation or "preloading" method is in general a more efficient method to encapsulate big macromolecules (She et al., 2010). Recently, it has been demonstrated that by conjugating $500 \mathrm{kDa}$ dextran to different ion-sensitive fluorophores, it is possible to load efficiently the microcapsules that otherwise are too porous to avoid the leaking out of the small analyte-sensitive fluorophores from the inner cavity of the carrier system. Following this procedure, microcapsules sensitive to ions such as $\mathrm{H}^{+}$, $\mathrm{Na}^{+}, \mathrm{K}^{+}, \mathrm{Cl}^{-}$can be obtained (del_Mercato et al., 2011). The main aim of such capsule-based sensors is to be able to estimate quantitatively the concentration of certain ions that are involved in intracellular processes. This objective has been achieved by characterizing the fluorescence response of ratiometric ion-sensitive polyelectrolyte capsules in which two fluorophores, one sensitive to a specific ion and one insensitive were entrapped in the same container. Again, by taking the ratio of the emission intensities at one single excitation wavelength, one can obtain an ion concentration value independent of the fluorophore concentration in a particular compartment. By titration fluorometric measurements and fluorescence microscopy it was possible to generate calibration curves that served to estimate ion concentration.

Very similar to the Debye-Hückel effect described for the nanoparticles, the Donnan effect can generate a different ion concentration between the environment and the interior of the capsule, where the sensor molecules are entrapped (Sukhorukov et al., 1999). However, this problem can be overcome by correlating the external concentration of ions with the global optical response of the capsule, independently of the ion concentration in the inner cavity. Capsules sensitive to $\mathrm{pH}$ have shown the highest efficiency in sensing since the fluorescence emission of the specific fluorophore was not disturbed by the presence of the charged dextran (to which the $\mathrm{pH}$-sensitive fluorophores has been linked in order to be big enough for being retained in the capsule volume) or the polyelectrolytes in the capsule wall (Rivera_Gil et al., 2012). In the case of $\mathrm{Na}^{+}-, \mathrm{K}^{+}$-, and $\mathrm{Cl}^{-}$-sensor capsules the efficiency of the ion sensing respect to the fluorophore alone decreased, but they still were useful to estimate the desired range of concentration relevant for bio-medical applications. Nevertheless, the versatility of the synthesis of these ratiometric ion-sensitive polyelectrolyte capsules is a promising procedure to design quantitative ion sensors based on organic fluorophores.

The particular novelty and the differences to other alternative technologies such as the probes encapsulated by biologically localized embedding (PEBBLE) system (Clark et al., 1998) is that our carrier-based optical sensors in principle allow multiplexed measurements (del Mercato et al., 2011). This is based on the fact that capsules can be functionalized with fluorescent molecules at two distinct positions, in their walls and in their cavities. In this concept, fluorophores sensitive to different analytes are loaded into the cavities of different capsules and the walls of each capsule is fluorescently labeled with a barcode (Abbasi et al., 2011; Figure 1B). The color of the capsule wall allows for the identification of each capsule thus providing information for which analyte this particular capsule is sensitive to. In conclusion, sensor based microcapsules offer a great possibility to engineer multidetection systems in which several intracellular ions could be sensed in parallel. On the other hand, the disadvantage of these systems compared to the nanoparticles is that the functionalization of the capsule surface is not well established yet and much work has to be done in order to tune the microcapsules to target them to specific cellular compartments.

Although many different types of cells internalized these carriers (Sukhorukov et al., 2005; Muñoz_Javier et al., 2008; Rivera_Gil et al., 2009; Reibetanz et al., 2010), the capsules have only been found in lysosomal structures so far (Reibetanz et al., 2007; De Koker et al., 2009), being the mechanism of internalization of polymeric capsules not yet elucidated. The lysomotropy showed by the capsules can be turned into an advantage. As their location is exactly defined, capsules can be used to measure changes in the concentration of lysosomal ions, e.g., $\mathrm{H}^{+}$in situ over long periods of time (Rivera_Gil et al., 2012), which can be beneficial for many different diseases associated with a defective lysosomal acidification (Stutts et al., 1995; Mall et al., 1996; Matsui et al., 1998; Darbha et al., 2007; Lang, 2007).

Summarizing, the main and distinctive advantage of using polymeric microcapsules as carriers for the analyte-sensitive fluorophores is multiplexing, i.e., the ability to measure several analytes in parallel with barcoded microcapsules-based sensors. Furthermore, due to their big size which corresponds mostly to the cavity area, higher quantities of sensor molecules can be encapsulated and thus, those signals are higher. Since the intracellular fate of these microcapsules is different as the nano-based sensors, information from different cellular structures can be obtained upon their use.

\section{INTEGRATION OF THE NANOSENSORS INTO THE MICROMETER-SIZED CAPSULES}

As presented here, the idea will be to have a complex tool kit for sensing different ions in different conditions and environments. Regarding biological/medical applications this is particularly interesting since many diseases are directly related with alterations in ion concentrations. To our knowledge, the concentration of ions inside some cellular compartments has not been determined at all. Incubation of the cells either with the nanoparticles or with the capsules lead to a rapid incorporation of both systems. However, due to differences in size as well as other physicochemical properties, those two systems are internalized through different mechanisms of internalization. Depending on the chemical composition of the surface (tuning abilities), nanoparticles are taken up mostly either via clathrin- or via lipid 
raft-mediated endocytic processes (Jiang et al., 2010). Once incorporated, the nanoparticles are located in different compartments and normally end up in the lysosome. On the contrary, the internalization processes of the microcapsules are more complicated and are not fully known yet. Thus, it might not involve clathrin, caveolae mechanisms, and their derived endosomal localization, since the size of these pits is too small to engulf the capsules. Nevertheless, the final location of these microcapsules seems to be also the lysosome as commented before. Although both systems end up in the lysosomes, the compartments that are involved in the internalization processes until they reach the lysosome are different. Furthermore, the maturation of the vesicles involves fusion of different compartments according to the mechanism of internalization. In this way by using either the nanosensors or the micro-sensors, each of these compartments can be resolved over time.

One of the biggest advantages of the microcapsules relies on the possibility to combine different functionalities at different positions of the capsules (cavity or wall) to create a more sophisticated system. As described before, fluorophores (such as colloidal quantum dots) emitting at different wavelengths can be added to the wall of the capsules, while the cavity is filled with the analyte-sensitive fluorophore. In this case multiplexing can be easily achieved since every sensor can be recognized by the color of its wall (Abbasi et al., 2011). Furthermore, the cavity of the microcapsules can be loaded with the nanoparticle-based sensors, whereas the wall can be functionalized with gold nanoparticles. In this way, light-responsive microcapsules (Skirtach et al., 2005) can be used as carriers to deliver the nanosensors into the cytosol of the cells. Gold nanoparticles are able to absorb light and convert it into heat (Dondapati et al., 2010; Hühn et al., 2012). Then, the light illumination of gold nanoparticles presented in the wall of the capsules produces local heat enough to open the capsule and release the cargo into the cytosol (Carregal Romero et al., 2012). Ideally, the nanoparticle-based sensors could additionally be functionalized with target moieties to recognize organelles like endoplasmatic reticulum, pre-synaptic vesicles, nucleus, etc., so that upon controlled release, the sensors are directed to specific organelles. The multi-compartmentalization of the capsules could

\section{REFERENCES}

Abbasi, A. Z., Amin, F., Niebling, T., Friede, S., Ochs, M., CarregalRomero, S., Martos, J. M. M., Rivera_Gil, P., Heimbrodt, W., and Parak, W. J. (2011). How colloidal nanoparticles could facilitate multiplexed measurements of different analytes with analyte-sensitive organic fluorophores. ACS Nano 5, $21-25$.

Alvarez-Puebla, R. A., ContrerasCaceres, R., Pastoriza-Santos, I., Perez-Juste, J., and Liz-Marzan, L. M. (2009). Au@pNIPAM colloids as molecular traps for surface-enhanced, spectroscopic, ultra-sensitive analysis. Angew. Chem. Int. Ed. Engl. 48, 138-143.
Aranzaes, J., Belin, C., and Astruc, D. (2006). Assembly of dendrimers with redox-active $[\{\mathrm{CpFe}(\mathrm{mu}(3)-$ $\mathrm{CO})\}(4)]$ clusters at the periphery and their application to oxo-anion and adenosine-5'-triphosphate sensing. Angew. Chem. Int. Ed. Engl. $45,132-136$.

Bakker, E., Buhlmann, P., and Pretsch, E. (1997). Carrier-based ion-selective electrodes and bulk optodes. 1. General characteristics. Chem. Rev. 97, 3083-3132.

Ballerstadt, R., and Schultz, J. (2000). A fluorescence affinity hollow fiber sensor for continuous transdermal glucose monitoring. Anal. Chem. 72, 4185-4192.

additionally be used to combine nano- and the micro-sensor into one single system (Figure 1C). Whereas the nanosensors could be conjugated to the wall of the capsules via PEGylation of the outermost layer of the capsule, the cavity could be loaded with a different analyte-sensitive fluorophore. In this way two different ions could be estimated at the same time. Possible differences on the loading efficiencies of the different sensors inside each individual cell would then be avoided. For example, the nanoparticles sensitive to chloride could be incorporated in the capsule wall, while the proton-sensitive fluorophores could be loaded inside the capsule. In this way, the wall of the capsules would respond to changes in the chloride concentration, whereas the cavity of the capsules would read-out changes of the $\mathrm{pH}$. Furthermore, conjugation of the nanosensors to the wall of the capsule would lead to a local accumulation of the sensors in the area, which in turn would increase the signal of the nanosensors. This would again solve problems derived from an inefficient loading of the nanoparticles inside the cells since if too few nanoparticles are incorporated, the read-out signal cannot be detected.

\section{FINAL REMARKS}

In conclusion, the presented biosensors based on nanoparticles or microscopic polymeric structures have been demonstrated to own a good signal-to-noise ratio. The read-out can easily be detected with simple, non-invasive techniques. Due to their small sizes they can be used for intracellular measurements. Furthermore, due to the high versatility of the microcapsules, more sophisticated sensors can be generated, which are suitable for multiplexing measurements. We would also like to remark, that nanoparticles and microcapsules do not necessarily compete against each other. They can either be integrated in one single system to combine functionalities or can be used complementary to obtain more information from the cell, since both can be located in different cellular regions.

\section{ACKNOWLEDGMENTS}

This work was supported by the European Commission (grant Nandiatream to Wolfgang J. Parak). Susana Carregal-Romero is grateful to the Junta Andalucía for her current PostDoctoral fellowship.

Becker, A. L., Johnston, A. P. R., and Caruso, F. (2010). Layer-by-layerassembled capsules and films for therapeutic delivery. Small 6, 18361852.

Brown, J. Q., and McShane, M. J. (2005). Core-referenced ratiometric fluorescent potassium ion sensors using self-assembled ultrathin films on europium nanoparticles. IEEE Sens. J. 5, 1197-1205.

Buhlmann, P., Pretsch, E., and Bakker, E. (1998). Carrier-based ion-selective electrodes and bulk optodes. 2. Ionophores for potentiometric and optical sensors. Chem. Rev. 98, 1593-1687.

Burns, A., Sengupta, P., Zedayko, T., Baird, B., and Wiesner, U.
(2006). Core/shell fluorescent silica nanoparticles for chemical sensing: towards singleparticle laboratories. Small 2, 723-726.

Cameron, I. L., Smith, N. K. R., Pool, T. B., and Sparks, R. L. (1980). Intracellular concentration of sodium and other elements as related to mitogenesis and oncogenesis in vivo. Cancer Res. 40, 1493-1500.

Carregal Romero, S., Ochs, M., Rivera_Gil, P., Gana, C., Pavlov, A. M., Sukhorukov, G. B., and Parak, W. J. (2012). NIR-light triggered delivery of macromolecules into the cytosol. J. Control. Release 159, 120-127. 
Chen, F. Q., and Gerion, D. (2004). Fluorescent CdSe/ZnS nanocrystalpeptide conjugates for long-term, nontoxic imaging and nuclear targeting in living cells. Nano Lett. 4, 1827-1832.

Choi, W. S., Koo, H. Y., Zhongbin, Z., Li, Y., and Kim, D.-Y. (2007). Templated synthesis of porous capsules with a controllable surface morphology and their application as gas sensors. Adv. Funct. Mater. 17, 1743-1749.

Chung, Y. I., Kim, J. C., Kim, Y. H., Tae, G., and Lee, S. Y., Kim, K., and Kwon, I. C. (2010). The effect of surface functionalization of PLGA nanoparticles by heparinor chitosan-conjugated Pluronic on tumor targeting. J. Control. Release 143, 374-382.

Clark, H. A., Barker, S. L. R., Brasuel, M., Miller, M. T., Monson, E., Parus, S., Shi, Z. Y., Song, A., Thorsrud, B., Kopelman, R., Ade, A., Meixner, W., Athey, B., Hoyer, M., Hill, D., Lightle, R., and Philbert, M. A. (1998). Subcellular optochemical nanobiosensors: probes encapsulated by biologically localised embedding (PEBBLEs). Sens. Actuators B Chem. 51, 12-16.

Cui, B. X., Wu, C. B., Chen, L., Ramirez, A., Bearer, E. L., Li, W. P., Mobley, W. C., and Chu, S. (2007). One at a time, live tracking of NGF axonal transport using quantum dots. Proc. Natl. Acad. Sci. U.S.A. 104, 13666-13671.

Darbha, G. K., Ray, A., and Ray, P. C. (2007). Gold nanoparticle-based miniaturized nanomaterial surface energy transfer probe for rapid and ultrasensitive detection of mercury in soil, water, and fish. ACS Nano 1, 208-214.

De, M., Ghosh, P. S., and Rotello, V. M. (2008). Applications of nanoparticles in biology. Adv. Mater. 20, $1-17$.

De Koker, S., De Geest, B. G., Singh, S. K., De Rycke, R., Naessens, T., Van Kooyk, Y., Demeester, J., De Smedt, S. C., and Grooten, J. (2009). Polyelectrolyte microcapsules as antigen delivery vehicles to dendritic cells: uptake, processing, and crosspresentation of encapsulated antigens. Angew. Chem. Int. Ed. Engl. 48, 8485-8489.

Debye, P., and Hückel, E. (1923a). Zur theorie der elektrolyte. Phys. Z. 24, 185-206.

Debye, P., and Hückel, E. (1923b). Zur theorie der elektrolyte ii. Das grenzgesetz für die elektrische leitfähigkeit. Phys. Z. 24, 305-325.

del Mercato, L. L., Abbasi, A. Z., Ochs, M., and Parak, W. J. (2011). Multiplexed sensing of ions with barcoded polyelectrolyte capsules. ACS Nano 5, 9668-9674.

Delcea, M., Yashchenok, A., Videnova, K., Kreft, O., Möhwald, H., and Skirtach, A. G. (2010). Multicompartmental micro- and nanocapsules: hierarchy and applications in biosciences. Macromol. Biosci. 10, 465.

del_Mercato, L. L., Abbasi, A. Z., and Parak, W. J. (2011). Synthesis and characterization of ratiometric ion-sensitive polyelectrolyte capsules. Small 7, 351-363.

De_Geest, B. G., De Koker, S., Sukhorukov, G. B., Kreft, O., Parak, W. J., Skirtach, A. G., Demeester, J., De Smedt, S. C., and Hennink, W. E. (2009). Polyelectrolyte microcapsules for biomedical applications. Soft Matter 5, 282-291.

De_Koker, S., De_Geest, B. G., Cuvelier, C., Ferdinande, L., Deckers, W., Hennink, W. E., De_Smedt, S., and Mertens, N. (2007). In vivo cellular uptake, degradation, and biocompatibility of polyelectrolyte microcapsules. Adv. Funct. Mater. 17, 3754-3763.

Dimosthenis, L. G., and Athanasios, G. V. (2011). Synthetic membranes (vesicles) in inorganic ion analysis: a review. Anal. Chim. Acta 683, 156-169.

Dolga, A. M., Terpolilli, N., Kepura, F., Nijholt, I. M., Knaus, H. G., D’orsi, B., Prehn, J. H., Eisel, U. L., Plant, T., Plesnila, N., and Culmsee, C. (2011). $\mathrm{KCa} 2$ channels activation prevents $\left[\mathrm{Ca}^{2+}\right] \mathrm{i}$ deregulation and reduces neuronal death following glutamate toxicity and cerebral ischemia. Cell Death Dis. 2, e147.

Donath, E., Sukhorukov, G. B., Caruso, F., Davis, S. A., and Möhwald, H. (1998). Novel hollow polymer shells by colloid-templated assembly of polyelectrolytes. Angew. Chem. Int. Ed. Engl. 37, 2202-2205.

Dondapati, S. K., Sau, T. K., Hrelescu, C., Klar, T. A., Stefani, F. D., and Feldmann, J. (2010). Label-free biosensing based on single gold nanostars as plasmonic transducers. ACS Nano 4, 6318-6322.

Dulkeith, E., Morteani, A. C., Niedereichholz, T., Klar, T. A., Feldmann, J., Levi, S. A., Van_Veggel, F. C. J. M., Reinhoudt, D. N., Möller, M., and Gittins, D. I. (2002). Fluorescence quenching of dye molecules near gold nanoparticles: radiative and nonradiative effects. Phys. Rev. Lett. 89, 203002-203001, 202002-203004.

Fu, Y., and Lakowicz, J. R. (2009). Modification of single molecule fluorescence near metallic nanostructures. Laser Photon. Rev. 3, 221-232.
Ge, Y. Q., Zhang, Y., Xia, J. G., Ma, M., He, S. Y., Nie, F., and $\mathrm{Gu}$, N. (2009). Effect of surface charge and agglomerate degree of magnetic iron oxide nanoparticles on $\mathrm{KB}$ cellular uptake in vitro. Colloids Surf. B Biointerfaces 73, 294-301.

Geiser, M., Rothen-Rutishauser, B., Kapp, N., Schurch, S., Kreyling, W., Schulz, H., Semmler, M., Hof, V. I. Heyder, J., and Gehr, P. (2005). Ultrafine particles cross cellular membranes by nonphagocytic mechanisms in lungs and in cultured cells. Environ. Health Perspect. 113, 1555-1560.

Grabchev, I., and Guittonneau, S. (2006). Sensors for detecting metal ions and protons based on new green fluorescent poly (amidoamine) dendrimers peripherally modified with 1,8-naphthalimides. J. Photochem. Photobiol. A Chem. 179, 28-34.

Grabchev, I., Soumillion, J. P., Muls, B., and Ivanova, G. (2004). Poly(amidoamine) dendrimer peripherally modified with $4-\mathrm{N}, \mathrm{N}$ dimethylaminoethyleneamino-1,8naphthalimide as a sensor of metal cations and protons. Photochem. Photobiol. Sci. 3, 1032-1037.

Hartig, S. M., Greene, R. R., Dikov, M. M., Prokop, A., and Davidson, J. M. (2007). Multifunctional nanoparticulate polyelectrolyte complexes. Pharm. Res. 24, 2353-2369.

Harush-Frenkel, O., Rozentur, E. Benita, S., and Altschuler, Y. (2008). Surface charge of nanoparticles determines their endocytic and transcytotic pathway in polarized MDCK cells. Biomacromolecules 9 , 435-443.

Hidalgo, G., Burns, A., Herz, E., Hay, A., Houston, P., Wiesner, U., and Lion, L. (2009). Functional tomographic fluorescence imaging of ph microenvironments in microbial biofilms by use of silica nanoparticle sensors. Appl. Environ. Microbiol. 75, 7426-7435.

Howarth, M., Liu, W. H., Puthenveetil, S., Zheng, Y., Marshall, L. F., Schmidt, M. M., Wittrup, K. D., Bawendi, M. G., and Ting, A. Y. (2008). Monovalent, reduced-size quantum dots for imaging receptors on living cells. Nat. Methods 5, 397-399.

Hühn, D., Govorov, A., Gil, P. R., and Parak, W. J. (2012). Photostimulated Au nanoheaters in polymer and biological media: characterization of mechanical destruction and boiling. Adv. Funct. Mater. 22, 294-303.

Jiang, W., Kim, B. Y. S., Rutka, J. T., and Chan, W. C. W. (2008). Nanoparticle-mediated cellular response is size-dependent. Nat. Nanotechnol. 3, 145-150.

Jiang, X. E., Dausend, J., Hafner, M., Musyanovych, A., Rocker, C., Landfester, K., Mailander, V., and Nienhaus, G. U. (2010). Specific effects of surface amines on polystyrene nanoparticles in their interactions with mesenchymal stem cells. Biomacromolecules 11, 748-753.

Kazakova, L. I., Shabarchina, L. I., and Sukhorukov, G. B. (2011). Coencapsulation of enzyme and sensitive dye as a tool for fabrication of microcapsule based sensor for urea measuring. Phys. Chem. Chem. Phys. 13, 11110.

Kolbe, A., Del Mercato, L. L., Abassi, A. Z., Rivera_Gil, P., Gorzini, S. J., Huibers, W. H. C., Poolman, B., Parak, W. J., and Herrmann, A. (2011). De Novo Design of supercharged, unfolded protein polymers, and their assembly into supramolecular aggregates. Macromol. Rapid Commun. 32, 186-190.

Kreft, O., Muñoz_Javier, A., Sukhorukov, G. B., and Parak, W. J. (2007). Polymer microcapsules as mobile local pH-sensors. J. Mater. Chem. 17, 4471-4476.

Lang, F. (2007). Mechanisms and significance of cell volume regulation. J. Am. Coll. Nutr. 26, 613S-623S.

Lin, C.-A. J., Sperling, R. A., Li, J. K., Yang, T.-Y., Li, P.-Y., Zanella, M., Chang, W. H., and Parak, W. J. (2008). Design of an amphiphilic polymer for nanoparticle coating and functionalization. Small 4, 334-341.

Mailander, V., and Landfester, K. (2009). Interaction of nanoparticles with cells. Biomacromolecules 10 , 2379-2400.

Mall, M., Hipper, A., Greger, R., and Kunzelmann, K. (1996). Wild type but not delta F508 CFTR inhibits $\mathrm{Na}+$ conductance when coexpressed in Xenopus oocytes. FEBS Lett. 381, 47-52.

Matsui, H., Grubb, B. R., Tarran, R., Randell, S. H., Gatzy, J. T., Davis, C. W., and Boucher, R. C. (1998). Evidence for periciliary liquid layer depletion, not abnormal ion composition, in the pathogenesis of cystic fibrosis airways disease. Cell 95 1005-1015.

McShane, M., and Ritter, D. (2010). Microcapsules as optical biosensors. J. Mater. Chem. 20, 8189-8193.

Muñoz_Javier, A., Kreft, O., Semmling, M., Kempter, S., Skirtach, A. G., Bruns, O., Pino, P. D., Bedard, M. F., Rädler, J., Käs, J., Plank, C., Sukhorukov, G., and Parak, W. J. (2008). Uptake of colloidal polyelectrolyte 
coated particles and polyelectrolyte multilayer capsules by living cells. Adv. Mater. 20, 4281-4287.

Nakai, T., Kanamori, T., Sando, S., and Aoyama, Y. (2003). Remarkably size-regulated cell invasion by artificial viruses. Saccharide-dependent self-aggregation of glycoviruses and its consequences in glycoviral gene delivery. J. Am. Chem. Soc. 125, 8465-8475.

Nativo, P., Prior, I. A., and Brust, M. (2008). Uptake and intracellular fate of surface-modified gold nanoparticles. ACS Nano 2, 1639-1644.

Nguyen, T., and Rosenzweig, Z. (2002). Calcium ion fluorescence detection using liposomes containing Alexalabeled calmodulin. Anal. Bioanal. Chem. 374, 69-74.

Osaki, F., Kanamori, T., Sando, S., Sera, T., and Aoyama, Y. (2004). A quantum dot conjugated sugar ball and its cellular uptake. On the size effects of endocytosis in the subviral region. $J$. Am. Chem. Soc. 126, 6520-6521.

Pellegrino, T., Manna, L., Kudera, S., Liedl, T., Koktysh, D., Rogach, A. L., Keller, S., Rädler, J., Natile, G., and Parak, W. J. (2004). Hydrophobic nanocrystals coated with an amphiphilic polymer shell: a general route to water soluble nanocrystals. Nano Lett. 4, 703-707.

Perez-Pineiro, R., Correa-Duarte, M. A., Salgueirino, V., and Alvarez-Puebla, R. A. (2012). SERS assisted ultrafast peptidic screening: a new tool for drug discovery. Nanoscale 4, 113-116.

Peteiro-Cartelle, J., Rodríguez-Pedreira, M., Zhang, F., Rivera_Gil, P., Del_Mercato, L. L., and Parak, W. J. (2009). One example on how colloidal nano- and microparticles could contribute to medicine. Nanomedicine 4, 967-979.

Reibetanz, U., Chen, M. H. A., Mutukumaraswamy, S., Liaw, Z. Y., Oh, B. H. L., Venkatraman, S., Donath, E., and Neu, B. R. (2010). Colloidal DNA carriers for direct localization in cell compartments by $\mathrm{pH}$ sensoring. Biomacromolecules 11, 1779.

Reibetanz, U., Halozan, D., Brumen, M., and Donath, E. (2007). Flow cytometry of HEK 293 T cells interacting with polyelectrolyte multilayer capsules containing fluoresceinlabeled poly(acrylic acid) as a $\mathrm{pH}$ sensor. Biomacromolecules 8, 1928-1933.

Riedinger, A., Zhang, F., Dommershausen, F., Röcker, C., Brandholt, S., Nienhaus, G. U., Koert, U., and Parak, W. J. (2010). Ratiometric optical sensing of chloride ions with organic fluorophore-gold nanoparticle hybrids: a systematic study of distance dependency and the influence of surface charge. Small 6, 2590-2597.

Rivera Gil, P., Del Mercato, L. L., DelPino, P., Munoz-Javier, A., and Parak, W. J. (2008). Nanoparticle-modified polyelectrolyte capsules. Nano Today 3, 12-21.

Rivera_Gil, P., Hühn, D., Del Mercato, L. L., Sasse, D., and Parak, W. J. (2010). Nanopharmacy: inorganic nanoscale devices as vectors and active compounds. Pharmacol. Res. 62, 115-125.

Rivera_Gil, P., Koker, S. D., De_Geest, B. G., and Parak, W. J. (2009). Intracellular processing of proteins mediated by biodegradable polyelectrolyte capsules. Nano Lett. 9, 4398-4402.

Rivera_Gil, P., Nazarenus, M., Ashraf, S., and Parak, W. J. (2012). pH sensitive capsules as intracellular optical reporters for monitoring lysosomal ph changes upon stimulation. Small 8, 943-948.

Rodriguez-Lorenzo, L., Krpetic, Z., Barbosa, S., Alvarez-Puebla, R. A., Liz-Marzan, L. M., Prior, I. A., and Brust, M. (2011). Intracellular mapping with SERS-encoded gold nanostars. Integr. Biol. (Camb.) 3, 922-926.

She, Z., Antipina, M. N., Li, J., and Sukhorukov, G. B. (2010). Mechanism of protein release from polyelectrolyte multilayer microcapsules. Biomacromolecules 11, 1241-1247.

Skirtach, A. G., Dejugnat, C., Braun, D., Susha, A. S., Parak, W. J., Möhwald, H., and Sukhorukov, G. B. (2005). The role of metal nanoparticles in remote release of encapsulated materials. Nano Lett. 5, 1371-1377.

Stuart, M., Huck, W., Genzer, J., Muller, M., Ober, C., Stamm, M.,
Sukhorukov, G., Szleifer, I., Tsukruk, V., Urban, M., Winnik, F., Zauscher, S., Luzinov, I., and Minko, S. (2010) Emerging applications of stimuliresponsive polymer materials. Nat. Mater. 9, 101-113.

Stutts, M. J., Canessa, C. M., Olsen, J. C., Hamrick, M., Cohn, J. A., Rossier, B. C., and Boucher, R. C. (1995). $\mathrm{Cftr}$ as a camp-dependent regulator of sodium-channels. Science 269, 847-850.

Sukhorukov, G. B., Brumen, M., Donath, E., and Mohwald, H. (1999) Hollow polyelectrolyte shells: exclusion of polymers and Donnan equilibrium. J. Phys. Chem. B 103, 6434-6440.

Sukhorukov, G. B., Donath, E., Davis, S., Lichtenfeld, H., Caruso, F., Popov, V. I., and Möhwald, H. (1998). Stepwise polyelectrolyte assembly on particle surfaces: a novel approach to colloid design. Polym. Adv. Technol. 9 759-767.

Sukhorukov, G. B., Rogach, A. L., Zebli, B., Liedl, T., Skirtach, A. G., Köhler, K., Antipov, A. A., Gaponik, N., Susha, A. S., Winterhalter, M., and Parak, W. J. (2005). Nanoengineered polymer capsules: tools for detection, controlled delivery and site specific manipulation. Small 1 , 194-200.

Suzuki, M., Husimi, Y., Komatsu, H., Suzuki, K., and Douglas, K. T. (2008). Quantum dot FRET biosensors that respond to $\mathrm{pH}$, to proteolytic or nucleolytic cleavage, to DNA synthesis, or to a multiplexing combination. J. Am. Chem. Soc. 130 5720-5725.

Tan, W. H., Shi, Z. Y., Smith, S., Birnbaum, D., and Kopelman, R. (1992). Submicrometer intracellular chemical optical fiber sensors. Science 258, 778-781.

Thanh, N., and Green, L. (2010). Functionalisation of nanoparticles for biomedical applications. Nano Today 5, 213-230.

Yakovlev, A. V., Zhang, F., Zulqurnain, A., Azhar-Zahoor, A., Luccardini, C., Gaillard, S., Mallet, J. M., Tauc, P., Brochon, J. C., Parak, W. J., Feltz, A., and Oheim, M. (2009). Wrapping nanocrystals with an amphiphilic polymer preloaded with fixed amounts of fluorophore generates fret-based nanoprobes with a controlled donor/acceptor ratio. Langmuir 25, 3232-3239.

Zhang, F., Ali, Z., Amin, F., Feltz, A., Oheim, M., and Parak, W. J. (2010). Ion and $\mathrm{pH}$ sensing with colloidal nanoparticles: influence of surface charge on sensing and colloidal properties. Chemphyschem 11 , 730-735.

Zhang, F., Lees, E., Amin, F., Rivera_Gil, P., Yang, F., Mulvaney, P., and Parak, W. J. (2011). Polymer-coated nanoparticles: a universal tool for biolabelling experiments. Small 7, 3113-3127.

Zhang, Q., Li, Y. L., and Tsien, R. W. (2009). The dynamic control of kissand-run and vesicular reuse probed with single nanoparticles. Science 323, 1448-1453.

Zhu, J., Li, J., Wang, A., Chen, Y., and Zhao, J. (2010). Fluorescence quenching of alpha-fetoprotein by gold nanoparticles: effect of dielectric shell on non-radiative decay. Nanoscale Res. Lett. 5, 1496-1501.

Conflict of Interest Statement: The authors declare that the research was conducted in the absence of any commercial or financial relationships that could be construed as a potential conflict of interest.

Received: 01 February 2012; accepted: 05 April 2012; published online: 26 April 2012.

Citation: Carregal-Romero S, Montenegro J-M, Parak WJ and Rivera_Gil $P$ (2012) Subcellular carrier-based optical ion-selective nanosensors. Front. Pharmacol. 3:70. doi: 10.3389/fphar.2012.00070

This article was submitted to Frontiers in Neuropharmacology, a specialty of Frontiers in Pharmacology.

Copyright (C) 2012 Carregal-Romero, Montenegro, Parak and Rivera_Gil. This is an open-access article distributed under the terms of the Creative Commons Attribution Non Commercial License, which permits non-commercial use, distribution, and reproduction in other forums, provided the original authors and source are credited. 